Review

\title{
New, exciting developments in experimental therapies in the early 21st century
}

\author{
Marek Los* \\ Interfaculty Institute of Biochemistry, Hoppe-Seyler-Str. 4/401B, Univ. Tübingen, Germany \\ BioApplications Enterprises, 34 Vanier Dr., Winnipeg, MB, Canada R2V2N6
}

\section{A R T I C L E I N F O}

\section{Article history:}

Received 6 October 2009

Received in revised form 8 October 2009

Accepted 8 October 2009

Available online 13 October 2009

\section{Keywords:}

Bystander effect

Epidermal growth factor

DNMT

Her2/neu

HDAC

RAGE

S100A8/A9

SDF-1

Single nucleotide polymorphism

\begin{abstract}
A B S T R A C T
The volume is dedicated to novel anticancer strategies. Our aim was to identify and cover novel, emerging anticancer approaches that will form the backbone of future, more efficient anticancer therapies. Beside classical "small molecule" pharmacologic approaches, or radiotherapy, the review introduces cancer stem cell, their markers involved in metastasizing, it covers various immunotherapeutic experimental treatment, inclusive anticancer cellular vaccines, as well as computational strategies aimed at modeling of cancer therapy or at least the intermolecular interactions between drug and the cellular target. Large portion of the volume is dedicated to various targeted anticancer approaches that involve either novel targets within cancer cells (i.e. endoplasmic reticulum and protein folding, or cell-cell adherence), or novel molecules like TRAIL and another human cytokine mda-7/IL-24, Brevinin-2R, viral proteins R4orf4, NS1, and apoptin, HAMLET, onconase, and other molecules. Significant part of the review is also dedicated towards targeting of receptor-initiated and intracellular kinase cascades that are often upregulated in various malignancies. We hope that the variety of topics highlighted in this volume will foster cross-discipline collaborations, so necessary for the development of novel therapeutics.
\end{abstract}

(c) 2009 Elsevier B.V. All rights reserved.

\section{Contents}

1. Introduction . . . . . . . . . . . . . . . . 1

2. Cancer stem cells . . . . . . . . . . . . . . . . . . . . . . . . . . . . . . . . . . . . . . . . . . . . . . . . . . . . . . . . .

3. Receptor-integrated and other kinases as therapeutic targets . . . . . . . . . . . . . . . . . . . . . . . . . . . . 2

4. Cancer immunotherapies . . . . . . . . . . . . . . . . . . . . . . . . . . . . . . . . . . . . 2

5. Ultrastructural and computational approaches in the development of novel anticancer drugs . . . . . . . . . . . . . . . . . . . 2

6. Genetic variability and epigenetic factors - their effects on cancer therapy . . . . . . . . . . . . . . . . . . . . . . . . . . . . 3

7. New developments and future perspectives in cancer radiotherapy . . . . . . . . . . . . . . . . . . . . . . . . . . . . 3

8. Selective targeting of cancer cells using available, naturally-occurring molecules and their derivatives . . . . . . . . . . . . . . . . . . . . 4

9. Epilogue. . . . . . . . . . . . . . . . . . . . . . . . . . . . . . . . . 5

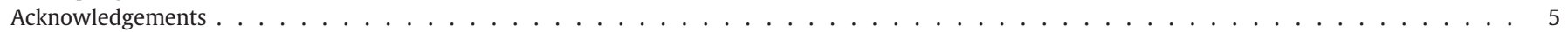

References. . . . . . . . . . . . . . . . . . . . . . . . . . . . . . . . . . . . . . . . . . 5

\section{Introduction}

Although the word cancer, describing malignant proliferatory diseases has been coined by ancient Greeks (i.e. Hippocrates refers to inflamed form of breast cancer this way) about 2500 years ago, current medicine still offers few curative treatments for most of the malignant diseases. This volume of The European Journal of

\footnotetext{
* BioApplications Enterprises, 34 Vanier Dr., Winnipeg, MB, Canada R2V2N6. Tel.: +1204 2279634.

E-mail address: bioappl@gmail.com.
}

Pharmacology is dedicated to highlighting some of the interesting new trends in the development of new experimental cancer therapies, as well as improvements to established therapies that are based on advances in our understanding of the patho-biology of cancer.

\section{Cancer stem cells}

Although the concept of "cancer-initiating-" or "cancer stem cells" has been around for about 40 years, only within the last decade this research area gained more attention, and with proof-of-principle experiments particularly in hematologic malignancies (Bonnet and 
Dick, 1997), it became more broadly accepted. Nowadays, cancer stem cells have been identified in most malignancies (Hombach-Klonisch et al., 2008; Klonisch et al., 2008) and attempts are being made to target them selectively. In this volume, Ratajczak et al. (2009-this volume) describes and highlights the role of Stromal-Derived Factor-1 (SDF-1) as an important regulator of trafficking of stem cells and possibly also cancer stem cells. SDF-1 is a chemokine that binds to the G-protein-coupled seven-transmembrane span CXCR4 and CXCR7. The SDF-1-CXCR4 axis regulates trafficking of normal and malignant cells. SDF-1 acts as an important chemoattractant for various cell types including hematopoietic stem/progenitor cells. Its newly discovered second receptor, CXCR7 may also be involved in several aspects of tumorogenesis and could become an important target for new anti-metastatic and anti-cancer drugs.

\section{Receptor-integrated and other kinases as therapeutic targets}

Growth of most cells in human body is regulated by coordinating signals transmitted mostly through cell-membrane receptors, and integrated kinase signaling cascades. The ErbB (Her) family of transmembrane tyrosine kinase (TK) receptors, regulate cellular processes such as cell growth, proliferation, survival, and differentiation. Members of the family include the Her1 (epidermal growth factor receptor, EGFR, ErbB1), Her2 (Her2/neu, ErbB2), Her3 (ErbB3) and Her4 (ErbB4). Thus, targeting Epidermal Growth Factor (EGF) dependent signaling pathways have been one of the success stories of modern oncology (Johnston et al., 2006). The therapeutic humanized monoclonal antibody Herceptin, (trastuzumab), a "small molecule" inhibitor Lapatinib (Tykerb), and other inhibitors of Her2 receptor signaling, have been among the most successful targeted therapeutics introduced to the clinic within the last decades. In this volume, two manuscripts, by Christoffersen et al. (2009-this volume) and Gadji et al. (2009-this volume), authors not only summarize our knowledge on targeting receptor-tyrosine kinase pathways, but also discuss important clinical problems and provide perspective view of the future development of this exciting and clinically very successful research field. While Gadji et al., focus on the EGF signaling, Christoffersen et al. provide a much broader perspective to the subject, discussing also intracellular tyrosine signaling cascades as therapeutic targets.

\section{Cancer immunotherapies}

Our immune system through scanning peptides presented on MHCmolecules, and by an array of receptors on cells within innate immunity compartment, constantly scrutinize our body for "non-self" molecular signatures. Clinical observations in immunosuppressed patients indicate that the immune system plays a major role in the elimination of emerging cancer cells, especially in its early stage. Thus, clinically emerging malignancy is a result of out-competition of the surveillance capacities of the immune system by cancer cells. Aggressive unspecific radio- and chemotherapies that target rapidly-dividing cells may in fact also disable specific antitumor immune response, by killing rapidly expanding specific immune-effector cells. Cancer immunotherapy, after a series of successes, about 100 years ago (i.e. "Coley's toxin"), is becoming again the major weapon against neoplastic malignancies. Among modern cancer immunotherapies, antibodytherapies (i.e. see above: Herceptin) have been clearly most widely, and most successfully used in modern clinical settings (Booy et al., 2006; Krzemieniecki et al., 2006). The manuscript by Borghaei et al. (2009-this volume), is dedicated to various immunotherapies of cancer, with the focus on antibody-therapies. Authors initially providing a historic perspective of immunotherapeutic approaches, and then they discuss various elements of the immune system in the context of their application for targeted antitumor therapies. They cover both the available "success stories" and experimental therapies that still await broad applications, and acceptance within medical community.
Among novel immunotherapeutic approaches, "arming" of antibodies in order to augment their toxicity, or using them as vectors to carry radioactive- or other type toxic payload (silver-bullet approach) is gaining popularity. Zoller et al. (2009-this volume), in this volume, discuss the principles, advantages and therapeutic potential of endoradiotherapy. The introduction to the subject is followed by some considerations on radio-biology (the interactions of various types of irradiation with the surrounding tissues), and its consequences for the choice of a radio-nuclide for a given target. Large part of the review is dedicated to the chemistry of direct and indirect labeling of the targeting vectors; also targeting principles are extensively discussed. Here, two major trends within the field, namely peptide-based targeting approaches, and antibody-immunotherapies are introduced. Authors also discuss the potentials, goals and avenues that the field of endo-radiotherapy will likely take follow in the near future.

The cytokine TRAIL emerges as one of the most-promising experimental cancer therapies. In this volume Holoch \& Griffith provide a historic perspective of the TRAIL research field, as well as review some current clinical trials that utilize TRIAL either as monotherapy, or in a combination with other anticancer treatments (Holoch and Griffith, 2009-this volume). TRAIL is a member of the tumor necrosis factor super family. It has been under intense research focus because of its remarkable ability to selectively induce apoptosis in malignant human cells. Many human tumor cells are capable of developing resistance to TRAIL-induced apoptosis, so a better understanding of underlying mechanisms may allow for the design of more effective therapies.

Components of calprotectin (S100A8/A9 protein complex) have gained a lot of attention in recent years, due to its modulatory activity on wound-healing and also due to the inhibitory activity of S100A9 towards antitumor immune response. Interestingly, the S100A8/A9 protein complex, at low concentration induces cell proliferation, in a RAGE- and MAP-kinase dependent manner (Ghavami et al., 2008c), whereas at a high micromolar concentration it induces a mixed (apoptotic and autophagic) form of cell death that involves lysosomes, and is modulated by BNIP3 (Ghavami et al., 2008b). In this volume, Ghavami and coauthors, cover not only pro-, and antiapoptotic functions of S100A8/A9, but also the immuno-regulatory activity of the protein complex, especially in relation to cancer, and its/their role in cancer spread (Ghavami et al., 2009-this volume). The last part of the review summarizes/recapitulates our knowledge about various functions of the S100A8/A9 complex.

Perhaps the most intriguing perspectives for cancer immunotherapies are in the field of cell-based anticancer immuno-vaccination. The brothers J. \& A. Mackiewicz introduce this interesting therapeutic concept that is already in clinical trials against melanoma and some other types of cancer (Mackiewicz and Mackiewicz, 2009-this volume). Anti-tumor vaccine development is a multi-step, complex process. It consists of two phases: preclinical and clinical. Guidelines on preclinical evaluation of cell based immunotherapy medicinal products have been clarified by regulatory agencies. However, the guidelines for clinical testing of therapeutic cancer vaccines, especially the end-points to consider, are currently hotly debated. Cancer vaccines are investigated in two types of clinical trials: proof-ofprinciple and efficacy. Readership interested in various aspects of clinical trial development are strongly encouraged to consult this article.

\section{Ultrastructural and computational approaches in the development of novel anticancer drugs}

Recent advances in high-resolution structural biology allow for deciphering of protein structures with very high accuracy. Such structural approaches are further enhanced by ever increasing computational power. Scientists deciphered to date the 3-dimensional 
structure of about 1000 proteins. Advancement in software combined with ever increasing computational power, and the availability of protein crystal structural "templates" allows further advances in modeling of structures of proteins that do not easily form crystals. Thus, some intermolecular interactions whose examination would previously be only possible in the tube, could actually be accurately modeled in silico.

In this volume, two papers describe ultra-structural approaches in the quest to develop novel anticancer therapeutics. Mandal et al. (2009this volume), discuss advantages and limitations of computer modeling of chemical structures and rationale drug design. Authors highlight various scenarios followed in order to develop (design in-silico) chemical structures with drug properties. They first discuss parameters that need to be considered when conceiving a new drug (i.e. chemical properties, stability, toxicity of its metabolites etc...). Among other approaches authors also highlight combined gene technology, and bioinformatics tools in rational drug design, and prevention of side effects (i.e. so called "ancient approach"). Finally authors discuss challenges and related to high-throughput screening approaches and the usefulness of bioinformatics and bio-statistic tools in that context.

Stetefeld and coworkers highlight a different approach. They describe the use of already available structural information, as well as the power of high resolution crystallographic methods to develop and refine nano-structures that could be in the future used as nanotransporters of anticancer drugs in order to improve their pharmacologic properties and diminish side-effects (McFarlane et al., 2009this volume). They focus on nano-structural tubes, so called coiled coil domains that harbor cavities and could easily be loaded with some common anticancer drugs (electrostatic principle). The authors provide the overview of the family of protein domains, its potential for drug packaging and drug delivery applications, and structural changes thereof upon loading. Finally, authors discuss future perspectives within the field and potential additional applications.

Computer sciences may be implemented into cancer therapy in an even more radical ways. In this volume, Swierniak et al. (2009-this volume) cover several major areas of mathematical modeling that may serve therapy optimization. Authors start with highlighting of early cell-cycle progression models, then they portrait techniques that take into account genetic aberrations and biologic consequences thereof. Then, they continue with more complex models involving evolution of drug resistance and pharmacokinetic and pharmacodynamic effects. Further parts of the review are dedicated to more recent areas, namely models involving angiogenesis of tumors and molecular signaling within, and between cells. Last part of the review is dedicated towards the "systems biology approach".

\section{Genetic variability and epigenetic factors - their effects on cancer therapy}

In the post-genome era where most cDNAs and their corresponding protein sequences could be calculated, the available information allows now to analyze genomic variabilities and sequence-neutral modifications within chromatin. Both single nucleotide polymorphism (SNP; a base substitution "mutation" found with the frequency higher than $1 \%$ within the genome) and chromatin modifications have sometimes dramatic effects on protein expression, and/or their activity. Those variables undoubtly affect both the diseases progression, and therapeutic outcome, since both the sensitivity to a given therapy as well as drug metabolism may be affected.

In this volume, Wiechec and Hansen (2009-this volume) paint the vision of future cancer therapy where the individual genomic profile of each patient will be first developed, leading to optimal personalized therapy, avoiding unnecessary toxicity, side effects or chemotherapeutic drugs for which the patient evolves resistance. The review is focused on SNPs, which are highly abundant in the genomes. Identification of specific SNP genotypes affecting transcriptional activity (and thereby the outcome for the patient) of genes involved in DNA repair, metabolizing of chemotherapeutic drugs and drugtargeted genes, leads to the development of personalized treatment of cancer. Authors highlight selected genes relevant primarily for breast cancer pathology, and use them as examples to explain the principles of medicine personalization.

In the article by Kristensen et al. (2009-this volume), authors review our current knowledge on common chromatin modifications, namely $\mathrm{CpG}$-metylations and histone acetylations. They also compare the differences between normal tissues and cancer, as well as discuss the involved enzymes and outline attempts to target them for anticancer therapy. In particular, they focus on DNA methyltransferases and histone deacetylases as epigenetic targets for cancer treatment. They also comment on synergistic effect of a combined use of DNMT and HDAC inhibitors. In the further part of the manuscript they discuss in detail sensitization of various cancers by epi-drugs to a large variety of conventional treatment strategies.

\section{New developments and future perspectives in cancer radiotherapy}

Interaction of charged particles, or high energy electromagnetic waves with the tissue, especially under sufficient oxygenation, leads to the occurrence of reactive oxygen species that in turn cause DNA strand breaks and other macromolecular changes. Sufficiently damaged genome is unable to repair itself and may ultimately lead to the death of the targeted cell. Irradiation through external sources, or by internal applications of radionuclides (brachytherapy) has been used for several years for cancer therapy, and is being constantly improved. More recently, antibody or peptide-directed delivery of radionuclides to tumor tissue (endoradiotherapy) has entered clinical testing phase with very promising results (Zoller et al., 2009-this volume).

Darzynkiewicz et al. (2009-this volume) provide a fresh look at the radiotherapy (and also chemotherapy) sensitivity in context of impaired DNA-damage response observed in many tumors. Authors provide a historic overview, and general information on the mechanisms of semi-selective action of DNA-damaging agents. This is followed by the detailed description of ATM-mediated response to DNA damage. Authors provide an overview of substrates phosphorylated by ATM, and the role of activation of checkpoint kinases and cell cycle arrest in this context. Next paragraph is dedicated to the relevant chromatin modifications, with the specific focus on histoneH2A phosphorylation. Finally, authors discuss the importance of impaired DNA-damage response for the selectivity of anticancer drugs that cause DNA damage, and indicate further possible approaches that would improve current anticancer therapies.

Following a similar principle, the DNA-repair machinery as a focus for the improvement of tumor radiotherapy sensitivity is also highlighted by Burdak-Rothkamm and Prise (2009-this volume). They propose targeting of DNA repair proteins using small molecule inhibitors, in combination with radiation and or chemotherapeutic agents as a means of enhancing cell killing. Authors discuss recent discoveries that may lead to the improvement of classical radio-and chemotherapies, by blocking molecules within different DNA-repair systems. The second part of the review is largely focused on the molecular mechanisms of radiation-induced bystander effect, and possible application of such knowledge both towards the improvement of therapy efficacy and prevention or weakening of side effects.

The radiation-triggered bystander effect is also in the focus of interest of Rzeszowska-Wolny et al. (2009-this volume). Authors discuss and summarize our current knowledge about various bystander effect mechanisms. Cells exposed to ionizing radiation show a broad spectrum of responses in cellular macromolecules, and for a long time it was generally accepted that effects such as DNA damage, apoptosis, chromosomal aberrations or increased mutation frequency resulted from ionization of cell structures and the action of reactive oxygen 
species formed by water radiolysis. In the last few years, however, it has appeared that cells exposed to ionizing radiation and other genotoxic agents can release signals that induce very similar effects in nontargeted neighboring cells, (bystander effects). These signals are conveyed to the neighboring non-hit cells by intercellular gap-junction communication or are released outside the cell, in the case of cultured cells into the medium. Interestingly, the bystander effects are not always cytotoxic, but they may also offer cytoprotection.

\section{Selective targeting of cancer cells using available, naturally-occurring molecules and their derivatives}

In recent years, several molecules have been identified that (semi) selectively kill cancer cells. Among them are already mentioned TRAIL (Holoch and Griffith, 2009-this volume) and another human cytokine mda-7/IL-24, Brevinin-2R (Ghavami et al., 2008a), viral proteins R4orf4, NS1, and apoptin (Maddika et al., 2008a; Maddika et al., $2008 \mathrm{~b}$ ), HAMLET ( $\alpha$-lactalbumin + oleic acid), onconase, and other molecules. Available data indicate that those molecules likely utilize different pathways to selectively kill cancer cells. This is a very optimistic observation because it assures the existence of several yetto be characterized common differences between normal and cancer cells that could be used for selective targeting of malignancies.

Mathew Noteborn highlights several of those molecules, while providing more detailed description of apoptin, a viral protein whose cancer-selective properties were originally described by his group in the last decade (Noteborn, 2009-this volume). Oncolytic viruses are known to replicate selectively in tumor cells resulting in cell death. Thus, some proteins derived from viruses (apoptin, E4orf4, NS1), all have the ability to induce tumor-selective apoptosis. These proteins seem to sense and modify transforming and survival processes, and redirect them into cell death pathways (Maddika et al., 2007). These proteins are also excellent indicator/sensor molecules for mapping differences between normal and transformed cells.

Wagstaff and Jans (2009-this volume) have been also analyzing the anticancer properties of apoptin, focusing however on its transformation-dependent nuclear transfer. They propose a modular, easy-adaptable system that would allow efficient delivery of therapeutic compounds, selectively to nuclei of cancer cells. Authors review problems associated with nuclear delivery of bioactive molecules, as well as recent progress towards the development of tumor-specific modular recombinant transporters as viable anti-cancer therapeutics.

Onconase is a small ribonuclease that exhibits strong, cancer(semi)selective toxicity (Ardelt et al., 2009-this volume). Darzynkiewicz and colleagues review our current knowledge on the use of RNases, especially onconase and related enzymes, as a novel form of protein-based anticancer drugs (biologics). Cytotoxic RNases are small $(10-28 \mathrm{kDa})$ basic proteins. They bind to negatively charged cell membrane, enter cells by endocytosis and translocate to cytosol where they evade mammalian protein ribonuclease inhibitor and degrade RNA. Onconase, the only enzyme of this class that reached clinical trials, is the smallest, stable, less catalytically efficient and more cytotoxic than most RNase-A homologues. Its cytostatic, cytotoxic and anticancer effects have been extensively studied. It targets rRNA, tRNA, mRNA as well as the non-coding RNA (microRNAs). Numerous cancer lines are sensitive to onconase; their treatment with $10-100 \mathrm{nM}$ onconase leads to suppression of cell cycle progression, predominantly through G1, followed by apoptosis or cell senescence.

Beside proteins, peptide-based approaches are becoming recognized as a novel and promising anticancer strategies. Most antimicrobial and anticancer cationic amphiphilic peptides share a common membranolytic mode of action. The electrostatic attraction between the negatively charged membrane components of bacterial and cancer cells and cationic amphiphilic peptides plays a crucial role in the disruption of bacterial and cancer cell membranes. Among these membranolytic cationic amphiphilic peptides, some clearly act independently of the membranolytic mecha- nism. Dr. Schweizer provides a historic perspective, and a comprehensive overview of the mode of action, and challenges that need to be overcome before cationic amphiphilic peptides will become widely used in the cancer therapy (Schweizer, 2009-this volume). He discusses both, membranolytic, and non-membranolytic mechanism of action these peptides, and furthermore, he highlights possible mechanisms responsible for cancer-selective action of some of these peptides. The development of cationic amphiphilic peptides-based chemotherapeutics is complicated by the traditionally poor pharmacokinetic properties and higher manufacturing costs of peptides, thus Dr. Schweizer discusses novel molecular, selective delivery devices that show promise in overcoming some of those phamacokinetic obstacles.

Short peptides and the respective peptidomometics are also in the center of attention of Devemy and Blaschuk (2009-this volume). Dr. Blaschuk has previously described short, cadherin-derived peptides capable of disrupting gap-junctions and selectively-target tumor vasculature. These discoveries and patents that followed, led to the foundation of Toronto-stock market-traded Adherex, a pharmaceutical company that develops novel antiangiogenic therapies. Authors review the role of type I and II superfamily of calcium-binding membrane glycoproteins, called cadherins in (tumor) blood vessel formation, in tumor metastasizing, and as a target for anticancer/ antivascular therapy. These cell adhesion molecules, link the microfilaments of the cytoskeleton through the interaction of their cytoplasmic domain with other elements of adherens junctions, and form intercellular adhesive complexes. They are crucial for holding cells together, and thus for tissue integrity. $\mathrm{N}$ - and VE-cadherin are key regulators of blood vessel formation and stability. Authors also discuss experiments showing that $\mathrm{N}$ - and $\mathrm{OB}$-cadherins facilitate cancer cell metastasis. Authors conclude that N- and VE-, and OBcadherin are attractive targets for cancer therapy.

Cancer cells with its fast metabolism require rapid turn-over of cellular proteins, thus ubiquitination pathway emerges as a very promising target for drug development (Ande et al., 2009-this volume). Maddika and colleagues review the different components of the ubiquitin network including E3 ligases, ubiquitin activating enzyme E1, proteasome and deubiquinylated enzymes that can be targeted for novel anticancer therapies. They also highlight "small molecule" inhibitors that have already been developed. The human ubiquitin system comprises hundreds of different enzymes including approximately 500 ubiquitin ligases, $\sim 50 \mathrm{E} 2 \mathrm{~s}$, and nearly 100 deubiquitinating enzymes. The majority of ubiquitin tagged proteins are channeled into a $26 \mathrm{~S}$ proteasome that consists of a catalytic $20 \mathrm{~S}$ core and the $19 \mathrm{~S}$ regulator. Together, these factors target thousands of human proteins for degradation or mediate other cellular functions. Many of these cellular functions are regulated by ubiquitin, and they are often deregulated in human cancers. Thus, due to abnormal expression or dysfunction of various ubiquitin components, ubiquitin system offers highly attractive drug targets for the development of novel and selective anti-cancer treatments.

Recent discovery of differential expression of some voltage-gated sodium channels opened a new and exciting path for the development of novel anticancer therapeutics (Onkal and Djamgoz, 2009-this volume). Authors discuss the potential of targeting of Voltage-Gated Sodium Channels, especially the neonatal Nav1.5, for metastatic breast cancer treatment. Inhibition of Voltage-Gated Sodium Channel either using the tetrodotoxin, gene silencing (siRNA) or a blocking polyclonal antibody, suppressed invasion, and directional motility, both integral to the metastatic cascade. On the contrary, transfecting a Voltage-Gated Sodium Channel into weakly invasive human prostate cancer cell line significantly increased invasiveness. Also, VoltageGated Sodium Channel expression has been positively correlated with metastatic status. It has been suggested that Voltage-Gated Sodium Channel upregulation is an early event in metastatic progression and that Voltage-Gated Sodium Channel expression is a 'switch', necessary and sufficient for engaging cancer cells in a highly invasive state. 
Like cancer immunotherapy, also some long-ago described molecules are awaking renewed interest as potential anticancer therapeutics. Solomon and Lee (2009-this volume), in this volume, focus on chloroquine and its derivatives as promising adjuvant agent for cancer therapy. The review provides both the historical perspective of chloroquine-related research as well as it discusses the development of various derivatives and their testing in clinical trials.

A lot of attention attracted within the last two decades apoptotic cell death induced either by triggering of surface receptors, or by activation of the mitochondrial death pathway. Healy et al. (2009-this volume) provide an overview of possible targets within endoplasmic reticulum as attractive and novel anticancer strategy. Authors extensively review the "unfolded protein response" and its role both in cancer progression and as a potential target for cancer therapies. In particular, they analyze the evidence suggesting that the ER stress response could be important for the growth and development of tumors under stressful conditions such as hypoxia or glucose deprivation, which are commonly encountered by most solid tumors. They also analyze possible ways to exploit the unfolded protein response as an anticancer strategy. Next, they discuss two approaches to target the unfolded protein response: (i) inhibiting components of the unfolded protein response so cells cannot adapt to stressful conditions, and (ii) overloading the unfolded protein response so the cell is unable to cope, thus leading to cell death. In the following part, they explore possibilities to target proteins with an enzymatic activity that could then be inhibited by "small molecule" drugs. Finally, the authors review drugs currently in clinical development that affect the ER stress response and that may have potential as anti-tumor agents alone or in combination with other chemotherapeutics.

\section{Epilogue}

We believe that modern pharmacological sciences have to partly break-away from tight focus on "small molecules" and instead explore an array of possibilities, cellular targets and novel vectors for drug delivery and even their targeting into specific cellular compartments. Thus, the volume is a collection of reviews from very diverse research areas bordering with classical pharmacology. We hope that illustration of various emerging approaches to target tumor biology will inspire the readership and result in cross-discipline pollination leading to the development of more effective anticancer therapies.

\section{Acknowledgements}

M.L. acknowledges the support from Deutsche Forschungsgemeinschaft (SFB 773, GRK 1302) and the Deutsche Krebshilfe.

\section{References}

Ande, S.R., Chen, J., Maddika, S., 2009. The ubiquitin pathway: An emerging drug target in cancer therapy. Eur. J. Pharmacol. 265, 199-205 (this volume).

Ardelt, W., Ardelt, B., Darzynkiewicz, Z., 2009. Ribonucleases as potential modalities in anticancer therapy. Eur. J. Pharmacol. 265, 181-189 (this volume).

Bonnet, D., Dick, J.E., 1997. Human acute myeloid leukemia is organized as a hierarchy that originates from a primitive hematopoietic cell. Nat. Med. 3, 730-737.

Booy, E.P., Johar, D., Maddika, S., Pirzada, H., Sahib, M.M., Gehrke, I., Loewen, S.D., Louis, S.D., Kadkhoda, K., Mowat, M., Los, M., 2006. Monoclonal and bispecific antibodies as novel therapeutics. Arch. Immunol. Ther. Exp. 54, 1-17.

Borghaei, H., Smith, M.R., Campbell, K.S., 2009. Immunotheraphy of Cancer. Eur. J. Pharmacol. 265, 41-54 (this issue)

Burdak-Rothkamm, S., Prise, K.M., 2009. New molecular targets in radiotherapy: DNA damage signalling and repair in targeted and nontargeted cells. Eur. J. Pharmacol. 265, 151-155 (this issue).

Christoffersen, T., Guren, T.K., Garm Spindler, K.-L., Dahl, O., Lønning, E., Tore Gjertsen, B., 2009. Cancer therapy targeted at cellular signal transduction mechanisms: strategies, clinical results, and unresolved issues. Eur. J. Pharmacol. 265, 6-22 (this volume).
Darzynkiewicz, Z., Traganos, F., Wlodkowic, D., 2009. Impaired DNA Damage Response - an Achilles' Heel Sensitizing Cancer to Chemotherapy and Radiotherapy. Eur. J. Pharmacol. 265, 143-150 (this issue).

Devemy, E., Blaschuk, O.W., 2009. Cadherins as novel targets for anti-cancer therapy. Eur. J. Pharmacol. 265, 195-198 (this volume).

Gadji, M., Tsanaclis Crous, A.M., Fortin, D., Krcek, J., Torchia, M., Mai, S., Drouin, R Klonisch, T., 2009. EGF receptor inhibitors in the treatment of glioblastoma multiform: old clinical allies and newly emerging therapeutic concepts. Eur. J. Pharmacol. 265, 23-30 (this volume).

Ghavami, S., Asoodeh, A., Klonisch, T. Halayko, A.J. Kadkhoda, K., Kroczak, T.J. Gibson, S.B Booy, E.P., Naderi-Manesh, H., Los, M., 2008a. Brevinin-2R(1) semi-selectively kills cancer cells by a distinct mechanism, which involves the lysosomal-mitochondrial death pathway. J. Cell. Mol. Med. 12, 1005-1022.

Ghavami, S., Chitayat, S., Hashemi, M., Eshraghi, M., Chazin, W.J., Halayko, A.J., Kerkhoff, C., 2009. S100A8/A9: a Janus-faced molecule in cancer therapy and tumorgenesis. Eur. J. Pharmacol. 265, 73-83 (this volume).

Ghavami, S., Kerkhoff, C., Chazin, W.J., Kadkhoda, K., Xiao, W., Zuse, A., Hashemi, M. Eshraghi, M., Schulze-Osthoff, K., Klonisch, T., Los, M., 2008b. S100A8/9 induces cell death via a novel, RAGE-independent pathway that involves selective release of Smac/DIABLO and Omi/HtrA2. Biochim. Biophys. Acta 1783, 297-311.

Ghavami, S., Rashedi, I., Dattilo, B.M., Eshraghi, M., Chazin, W.J., Hashemi, M., Wesselborg, S., Kerkhoff, C., Los, M., 2008c. S100A8/A9 at low concentration promotes tumor cell growth via RAGE ligation and MAP kinase-dependent pathway. J. Leukoc. Biol. 83, 1484-1492.

Healy, S.J.M., Gorman, A.M., Mousavi-Shafaei, P., Gupta, S., Samali, A., 2009. Targeting the endoplasmic reticulum-stress response as an anti-cancer strategy. Eur. J. Pharmacol. 265, 234-246 (this volume).

Holoch, P., Griffith, T.S., 2009. TNF-related apoptosis-inducing ligand (TRAIL): A new path to anti-cancer therapies. Eur. J. Pharmacol. 265, 63-72 (this volume).

Hombach-Klonisch, S., , 2009. Epigenetics and cancer treatment. Eur. J. Pharmacol. 265, 131-142 (this volume).

Krzemieniecki, K., Szpyt, E., Rashedi, I., Gawron, K., Los, M., 2006. Targeting of solid tumors and blood malignancies by antibody-based therapies. Centr. Eur. J. Biol. 1, 167-182.

Mackiewicz, J., Mackiewicz, A., 2009. Design of clinical trials for therapeutic cancer vaccines development. Eur. J. Pharmacol. 265, 84-89 (this volume).

Maddika, S., Ande, S.R., Panigrahi, S., Paranjothy, T., Weglarczyk, K., Zuse, A., Eshraghi, M., Manda, K.D., Wiechec, E., Los, M., 2007. Cell survival, cell death and cell cycle pathways are interconnected: Implications for cancer therapy. Drug Resist. Updat. $10,13-29$.

Maddika, S., Ande, S.R., Wiechec, E., Hansen, L.L., Wesselborg, S., Los, M., 2008a. Aktmediated phosphorylation of CDK2 regulates its dual role in cell cycle progression and apoptosis. J. Cell Sci. 121, 979-988.

Maddika, S., Wiechec, E., Ande, S.R., Poon, I.K., Fischer, U., Wesselborg, S., Jans, D.A., Schulze-Osthoff, K., Los, M., 2008b. Interaction with PI3-kinase contributes to the cytotoxic activity of apoptin. Oncogene 27, 3060-3065.

Mandal, S., Moudgil, M., Mandal, S., 2009. Rational drug design. Eur. J. Pharmacol. 265, 90-100 (this volume).

McFarlane, A., Orriss, G.L., Stetefeld, J., 2009. The use of coiled-coil proteins in drug delivery systems. Eur. J. Pharmacol. 265, 101-107 (this volume).

Noteborn, M.H.M., 2009. Proteins selectively killing tumor cells. Eur. J. Pharmacol. 265, 165-173 (this volume).

Onkal, R., Djamgoz, M.B.A., 2009. Molecular pharmacology of voltage-gated sodium channel expression in metastatic disease: clinical potential of neonatal Nav1.5 in breast cancer. Eur. J. Pharmacol. 265, 206-219 (this volume).

Ratajczak, M., Maksym, B.R., Grymula, K., Tarnowska, J., Wysoczynski, M., Liu, R., Ratajczak, J., Kucia, M., 2009. The Role of Stromal Derived Factor-1 - CXCR7 Axis in Development and Cancer. Eur. J. Pharmacol. 265, 31-40 (this volume).

Rzeszowska-Wolny, J., Przybyszewski, W.M., Widel, M., 2009. Ionizing radiationinduced bystander effects, potential targets for modulation of radiotherapy. Eur. J. Pharmacol. 265, 156-164 (this volume).

Schweizer, F., 2009. Cationic amphiphilic peptides (CAPs) with cancer-selective toxicity. Eur. J. Pharmacol. 265, 190-194 (this volume).

Solomon, R.V., Lee, H., 2009. Chloroquine and its analogs: a new promise of an old drug for effective and safe cancer therapies. Eur. J. Pharmacol. 265, 220-233 (this volume).

Swierniak, A., Kimmel, M., Smieja, J., 2009. Mathematical modeling as a tool for planning anticancer therapy. Eur. J. Pharmacol. 265, 108-121 (this volume).

Wagstaff, K.M., Jans, D.A., 2009. Nuclear drug delivery to target tumour cells. Eur. J. Pharmacol. 265, 174-180 (this volume).

Wiechec, E., Hansen, L.L., 2009. The effect of genetic variability on drug response in conventional breast cancer treatment. Eur. J. Pharmacol. 265, 122-130 (this volume).

Zoller, F., Eisenhut, M., Haberkorn, U., Mier, V., 2009. Endoradiotherapy in cancer treatment basic concepts and future trends. Eur. J. Pharmacol. 265, 55-62 (this volume). 